\title{
A robust approach for surface defect detection based on one dimensional local binary patterns
}

\author{
Shervan Fekri-Ershad and Farshad Tajeripour \\ Department of Computer Science, Engineering and IT, Shiraz University, Shiraz, Iran \\ shfekri@shirazu.ac.ir·tajeri@shirazu.ac.ir
}

\begin{abstract}
Defect detection is one of the problems in image processing and many different methods based on texture analysis have been proposed. The two dimensional local binary pattern approach provides discriminate features for texture analysis. In this paper for the first time, a method is proposed for detecting abnormalities in surface textures based on single dimensional local binary patterns. The proposed approach includes two steps. Firstly, in training step, single dimensional local binary patterns is applied on full defect-less surface images and the basic feature vector is calculated. Then, by image windowing and computing the non-similarity amount between these windows and basic vector, a threshold is computed for defect-less surfaces. Finally, in testing step, by using the defect-less threshold the defects are detected on test images. High detection rate, and low computational complexity are advantages of the proposed approach. The proposed approach is fully automatic and all of the necessary parameters can be tuned.
\end{abstract}

Keywords: Defect Detection, Image Processing, Feature extraction, Local Binary Patterns, Logarithm Likelihood Ratio Introduction

Visual quality inspection systems (VQIS) play an important role in many industrial and commercial applications such as tiles, metal, agricultural products, fabric, ceramic, paper and etc. Any hole, damage, abnormalities and slot in products surfaces are called defect. Ghazini et al. (2009) proposed a defect detection approach of tiles using combination of two dimensional wavelet transform and statistical features. Henry et al. (2006) used ellipsoidal region features and min-max technique on patterned fabric for detecting defects. Ch. Lin et al., (2009) described a texture defect detection system based on image deflection compensation. Tolba (2011) used a probabilistic neural network (PNN) for fast defect classification based on the maximum posterior probability of the Log-Gabor based statistical features. Alimohammadi et al., (2006) proposed a new method using optimal Gabor filters to detecting skin defect of fruits which was usable in agricultural products visual quality inspection systems (APVQIS). Some of defect detection approaches are compared by Xie et al. (2008).

The computational complexity of most of previous approaches is too high and some of them don't guarantee an accurate result for every surfaces and models of defects. So in this article, an approach is proposed to defect detection without these mentioned problems.

The Local binary patterns (LBP) is a non-parametric operator which describes the local spatial structure and local contrast of an image. It's first proposed by Ojala et al. (2000) and further improved by Ojala et al. (2002) which is a two dimensional operator.

In this paper first of all, a new version of LBP is proposed based on one dimensional modified local binary patterns for texture analyzing. Next, a novel approach is proposed for detecting surface defects based on one dimensional LBP. The proposed approach includes two steps. The first step is training. In this step, some surely defect-less images were taken and analyzed by one dimensional Local binary patterns (1DLBP) operator and a basic feature vector is computed, Which is a good identification for non-defects images. After that, by using image windowing technique and Log-Likelihood ratio, an accurate threshold is computed for defect-less image. The second step is testing. In this, step by extracting modified local binary patterns features of test images and compared them with basic feature vector, defect parts are detected. In the result part, some of fabric and stone images are provided from popular databases and the proposed approach applied on them. The fabric images are provided by department of electronic and electric of Hong-Kong University and the stone images are captured by digital camera. High detection rate shows the quality of the proposed approach. Low computation complexity, rotation invariant, and illumination invariant, are some of other advantages of proposed approach. Two dimensional Local binary patterns (2DLBP) and 1DLBP are compared in terms of detection rate and computational complexity.

\section{Local Binary Patterns}

The local binary patterns (LBP) is a non-parametric operator which describes the local contrast and local spatial structure of an image. For first time Ojala, et al. (2000) introduced this operator and showed its high severability and discriminative power for texture analyzing and classification.

In order to evaluate the LBP, at a given pixel position $\left(x_{c}, y_{c}\right)$, LBP is defined as an ordered set of binary comparisons of pixel intensities between the center pixel and its surrounding pixels. Usually to achieve the rotation invariant, neighborhoods would be assumed circular. So, points which the coordination's are not exactly located at the center of pixel would be found by interpolation. Some of the circular neighborhoods by different radius $(R)$ and $(P)$ number of neighborhoods pixels are shown in Fig.1. Now, the LBP are defined at a neighborhood of image by Eq.1.

Research article

CCIndian Society for Education and Environment (iSee)
"Defect detection"

http://www.indjst.org
S.F-Ershad \& F.Tajeripour Indian J.Sci.Technol. 
Fig 1. Some examples of circular neighborhoods (a) $P=8 R=1$

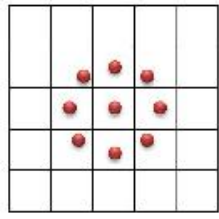

(a) (b) $P=12 R=1.5$

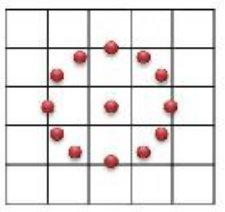

(b) (c) $P=16 \quad R=2$

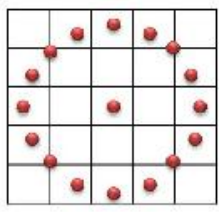

(c)
$\mathbf{L B P}_{\mathbf{P}, \mathbf{R}}=\sum_{\mathrm{k}=1}^{\mathrm{p}} \phi\left(\mathrm{g}_{\mathrm{k}}-\mathrm{g}_{\mathrm{c}}\right) 2^{\mathrm{k}-1}$

Where, "g $g_{c}$ " corresponds to the grey value of the centered pixel and " $\mathrm{g}_{\mathrm{k}}$ " to the grey values of the neighborhood pixels. So, $P$ will be the number of neighborhoods of center pixel, and function $\phi(x)$ is defined as:

$$
\phi(x)=\left\{\begin{array}{llr}
1 & \text { if } \quad x \geq 0 \\
0 & \text { else }
\end{array}\right.
$$

An example of applying $\operatorname{LBP}_{8,1}$ operator is shown in Fig.2. Where $\mathrm{LBP}_{8,1}$ amount of the center pixel is computed.

Fig. 2. An example of $\angle B P_{8,1}$ texture model

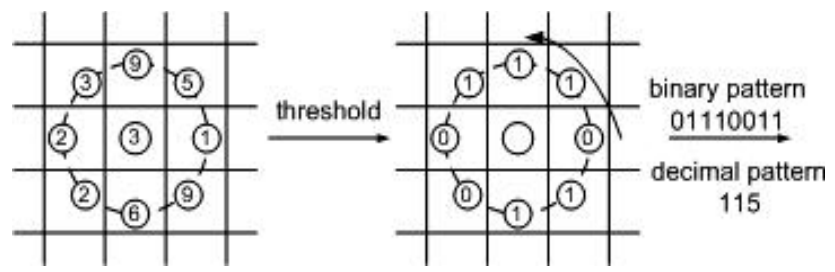

Achieving Rotation Invariance

According to (Ojala, et al., 2000), The $\mathrm{LBP}_{\mathrm{P}, \mathrm{R}}$ operator produces $\left(2^{P}\right)$ different output values, corresponding to the $2^{\mathrm{P}}$ different binary patterns that can be formed by the $\mathrm{P}$ pixels in the neighbor set. When the image is rotated, the gray values " $g_{k}$ " will correspondingly move along the perimeter of the circle around " $\mathrm{g}_{\mathrm{c}}$ ". To remove the effect of rotation, i.e. to assign a unique identifier to each rotation invariant local binary patterns is defined:

$$
\mathbf{L B P} P_{P, R}^{r i}=\min \left\{\operatorname{ROR}\left(\operatorname{LBP}_{P, R}, \beta\right) \mid \beta=0,1, \ldots, p-1\right\}
$$

Where "ri" correspond to rotation invariant and $\operatorname{ROR}(x, \beta)$ performs a circular bit-wise rotate right on the $\mathrm{P}$-bit number $x, \beta$ times. Finally, the minimum of computed values for $\beta=0$ to $p-1$ would be choose.

\section{Modified Two Dimensional Local Binary Patterns}

Practical experience of the authors (Pietikäinen et al., 2000), however, has shown that LBPROT as such does not provide very good discrimination, and the computation complexity of basic LBP is high. To solve these problems, Ojala, et al. (2002) defined a uniformity measure "U", which corresponds to the number of spatial transitions (bitwise 0/1 changes) in the "pattern". It is shown in Eq.4. For example, patterns 00000000 and
11111111 have $U$ value of 0 , while 11001001 have $U$ value of 3 .

$\mathbf{U}\left(\mathbf{L B P}_{\mathbf{P}, \mathbf{R}}\right)=\left|\phi\left(\mathrm{g}_{1}-\mathrm{g}_{\mathrm{c}}\right)-\phi\left(\mathrm{g}_{\mathrm{p}}-\mathrm{g}_{\mathrm{c}}\right)\right|+\sum_{\mathrm{k}=2}^{\mathrm{p}} \mid \phi\left(\mathrm{g}_{\mathrm{k}}-\right.$ $\mathrm{gc}-\phi(\mathrm{gk}-1-\mathrm{gc})$

In this version of LBP, the patterns which have uniformity amount less than $U_{T}$ are grouped as uniform patterns and the patterns with uniformity amount more than $U_{T}$ grouped as non-uniform patterns. Finally, the LBP is computed by using Eq. 5 .

$$
\mathbf{L B P}_{\mathrm{P}, \mathbf{R}}^{\mathrm{riu_{T }}}= \begin{cases}\sum_{\mathrm{k}=1}^{\mathrm{P}} \phi\left(\mathrm{g}_{\mathrm{k}}-\mathrm{g}_{\mathrm{c}}\right) & \text { if } \mathrm{U}\left(\mathrm{LBP}_{\mathrm{P}, \mathrm{R}}\right) \leq \mathrm{U}_{\mathrm{T}} \\ \mathrm{P}+1 & \text { elsewhere }\end{cases}
$$

Superscript "riu ${ }_{T}$ " reflects the use of rotation invariant "uniform" patterns that have $U$ value of at most $U_{T}$.

According to Eq.5, applying LBP will assign a label from 0 to $P$ to uniform patterns and label $P+1$ to non-uniform patterns. Because, 1DLBP just one label $(P+1)$ is assigned to all of the non-uniform patterns, so uniform labels should cover mostly patterns in the image. Tajeripour et al. (2008) showed that if in the definition of LBP operator the value of $U_{T}$ is selected equal to $(P / 4)$, only a negligible portion of the patterns in the texture takes label $\mathrm{P}+1$.

According to selecting two dimensional neighbors, the improved version of local binary patterns is a two dimensional operator which calls two dimensional local binary patterns (2DLBP). So, it causes much computation complexity for inspection systems. In the next section, one dimensional Local binary patterns (1DLBP) is proposed to decrease the computation complexity and increase the accuracy rate of texture analyzing and classification.

\section{Proposed Texture Analysis Operator}

In the basic LBP and 2DLBP operator which are described in sections 2 and 2.2, selecting neighborhood in circular form is to make the algorithm invariant to rotation. Since during visual quality inspection systems (VQIS) process, selecting circular neighborhood is not necessary. Also, computing brightness using

\begin{tabular}{|c|c|c|c|c|c|c|c|c|c|c|}
\hline g1 & $g^{2}$ & 83 & $\varepsilon^{4}$ & g5 & g6 & g7 & g8 & \multicolumn{3}{|c|}{ Row Segment Neighbors } \\
\hline 23 & 121 & 182 & 6 & 87 & 65 & 1 & 190 & 32 & 12 & Image Sample Row \\
\hline & 23 & 121 & 182 & 6 & 87 & 65 & 1 & 190 & Row & iegment \\
\hline & & 1 & 1 & 0 & 1 & 1 & 0 & 1 & 1DL & PApplied \\
\hline & 121 & 182 & 6 & 87 & 65 & 1 & 190 & 32 & Row & Segment \\
\hline & & 1 & 0 & 0 & 0 & 0 & 1 & 0 & 1DL & PApplied \\
\hline
\end{tabular}

Fig.3. Applying 1DLBP on a sample row of image with a segment of length 8 pixels 
interpolation in circular neighborhood needs a large amount of operations such as multiplication and additions which increase the computational complexity. Therefore, in this version of LBP, the neighborhood is a row (column) wise line segment. In order to apply one dimensional Local binary patterns (1DLBP), the gray value of the first pixel in the segment is compared with gray value of other pixels in the segment. Fig. 3 describes how to apply the 1DLBP operator on a sample column segment.

In 1DLBP, the uniformity measure "U" corresponds to the number of spatial transitions (bitwise 0/1 changes) in the row (column) segment (Eq.6).

$$
\begin{aligned}
& \mathbf{U}\left(\mathbf{L B P}_{\mathbf{L}}\right)=\mid \phi\left(\mathrm{g}_{\mathrm{L}}-\mathrm{g}_{1}\right)-\phi\left(\mathrm{g}_{2}-\mathrm{g}_{1}\right) \\
& +\sum_{\mathrm{k}=2}^{\mathrm{L}-1} \mid \phi\left(\mathrm{g}_{\mathrm{k}}-\mathrm{g}_{1}\right)-\phi\left(\mathrm{g}_{\mathrm{k}+1}-\mathrm{g}_{1}\right)
\end{aligned}
$$

Where $g_{k}$ represents the intensity of the $i_{t h}$ neighbors and $g_{1}$ is the intensity of the first pixel of each row (column) segment. Also, $L$ is the size of row (column) segments, so the notation of Local binary patterns is renamed from $\mathrm{LBP}_{\mathrm{P}, \mathrm{R}}$ to $\mathrm{LBP}_{\mathrm{L}}$.

In 1DLBP, row (column) segments that have uniformity amount less than uniformity threshold are categorized as uniform segments and row (column) segments that have uniformity amount more than uniformity threshold are categorized as non-uniform segments. So, the local binary patterns is measured by using Eq.7.

$$
\mathbf{L B P}_{\mathrm{L}}^{\mathrm{U}_{\mathrm{T}}}=\left\{\begin{array}{cc}
\sum_{\mathrm{k}=2}^{\mathrm{L}} \phi\left(\mathrm{g}_{\mathrm{k}}-\mathrm{g}_{1}\right) & \text { if } \mathrm{U}\left(\mathrm{LBP}_{\mathrm{L}}\right) \leq \mathrm{U}_{\mathrm{T}} \\
\mathrm{L} & \text { elsewhere }
\end{array}\right.
$$

According to Eq.8, if the length of the row (column) segment is considered $L$ pixels, applying $L B P_{L}$ will assign a label from 0 to $L-1$ to uniform segments and label $L$ to non-uniform segments.

Because, in 1DLBP just one label (L) is assigned to all of the non-uniform segments, so uniform labels should cover mostly segments. Tajeripour et al. (2008) showed that if in the definition of $L B P_{L}$ operator the value of $U_{T}$ is selected equal to $(L / 4)$, only a negligible portion of the patterns in the texture takes label $\mathrm{L}$.

According to selecting row (column) segments as neighbors, this version of local binary patterns is a one dimensional operator. So, its computation complexity is less than 2DLBP.

\section{Proposed Feature Vector}

As it was described in section 3 , a label is assigned to each row (column) segment. Regarding the Eq.7, if the length of each row (column) segment considered "L" pixels, applying $\mathrm{LBP}_{\mathrm{L}}$ will assign a label from zero to "L-1" to uniform segments and label "L" to non-uniform segments. So, for every image, two " $L+1 "$ dimensional feature vector can be extracted.

To extract the feature vector, first the $L_{B P}$ is applied on the image and the labels are assigned to row (column) segments. Then the occurrence probability of each label in the image is regarded as one of the dimensions of the feature vector. The occurrence probability of a specific label in the image can be approximated by the ratio of the number of that label to the number of all labels (Eq.8).

$$
\mathbf{P}_{\mathbf{k}}=\frac{\mathrm{N}_{\mathrm{P}_{\mathrm{k}}}}{\mathrm{N}_{\text {total }}} \quad 0 \leq \mathrm{k} \leq \mathrm{L}
$$

Where, $\mathrm{N}_{\mathrm{p}_{\mathrm{k}}}$ is the number of row (column) segments that labeled as $P_{k}$, and $N_{\text {total }}$ is the number of all row (column) segments. The feature extraction algorithm can be done once for the row segments and once for the column segments. So, for every image, two " $L+1$ " dimensional feature vector can be extracted. The extracted feature vector for row (column) segments is shown in Eq.9, and 10.

$\mathbf{R}_{\mathbf{x}}=\left\langle\mathrm{P}_{\mathrm{X}_{0}}, \mathrm{P}_{\mathrm{X}_{1}}, \ldots, \mathrm{P}_{\mathrm{X}_{\mathrm{L}}}\right\rangle$

$\mathbf{R}_{\mathbf{y}}=\left\langle\mathrm{P}_{\mathrm{y}_{0}}, \mathrm{P}_{\mathrm{y}_{1}}, \ldots, \mathrm{P}_{\mathrm{y}_{\mathrm{L}}}\right\rangle$

Where, $R_{x}$ is the feature vector extracted for row segmentations and $P x_{i}$ is the occurrence probability of label " $i$ " in row segments. There is a same way for $R_{y}$ and Py.

\section{Proposed Defect Detection Approach}

Any hole, damage, and abnormalities in surfaces are called defect. In this section, a general novel approach is proposed for detecting defects in surfaces. The proposed approach is a general one which is independent of texture analysis operator types. It is including of a training step and a testing step.

\section{Training Step}

In the training step, first an image is taken from the surface which is defect-less. Then texture analysis operator applied over the whole image. In this paper, 1DLBP is used. So, after that, regarding the Eq.9 and 10, two feature vectors are extracted from it. These vectors are called Basic feature vectors for rows and columns, and are marked by " $\mathrm{M}_{\mathrm{x}}$ " and " $\mathrm{M}_{\mathrm{y}}$ ".

Then the defect-less image is divided into windows of sizes $W \times W$ pixels. After that, the 1DLBP is applied over each of these windows. Thus, for each window, a feature vector is extracted for row (column) segments.

Then, the non-similarity amount of the rows (columns) vector in each window is computed through the basic rows (columns) feature vector based on Log-likelihood ratio (Eq.11, Eq.12).

$$
\begin{aligned}
& \mathbf{L}_{\mathrm{xi}}= \\
& \left(\mathrm{S}_{\mathrm{xi}}, \mathrm{M}_{\mathrm{x}}\right)=\sum_{\mathrm{k}=1}^{\mathrm{L}+1} \mathrm{~S}_{\mathrm{kxi}} \log \left(\frac{\mathrm{S}_{\mathrm{kxi}}}{\mathrm{M}_{\mathrm{xk}}}\right) \quad \mathrm{i}=1,2, \ldots, \mathrm{N} \\
& \mathbf{L}_{\mathrm{yi}}= \\
& \left(\mathrm{S}_{\mathrm{yi}}, \mathrm{M}_{\mathrm{y}}\right)=\sum_{\mathrm{k}=1}^{\mathrm{L}+1} \mathrm{~S}_{\mathrm{kyi}} \log \left(\frac{\mathrm{S}_{\mathrm{kyi}}}{\mathrm{M}_{\mathrm{yk}}}\right) \mathrm{i}=1,2, \ldots, \mathrm{N}
\end{aligned}
$$

Where " $S_{x i}=1$ and " $S_{y i}$ "is the feature vectors which extracted for " $\mathrm{i}_{\text {th }}$ " window in row wise and column wise. $M_{x}$ and $M_{y}$ are the basic feature vectors. Also, $N$ is the number of windows and "i" represents the " $\mathrm{K}_{\mathrm{th}}$ " dimension of the feature vector. 
Since minimization of Log-likelihood ratio shows the similarity to specific class. So, the maximum value among these ratios is regarded as the threshold for the defect-less window. (Eq.13, Eq.14)

$\mathbf{T}_{\mathbf{x}}=\max \left(\mathrm{L}_{\mathrm{xi}}\right) \quad \mathrm{i}=1,2, \ldots, \mathrm{N}$

$\mathbf{T}_{\mathbf{y}}=\max \left(\mathrm{L}_{\mathrm{yi}}\right) \quad \mathrm{i}=1,2, \ldots, \mathrm{N}$

Where, " $T_{x}$ " and " $T_{y}$ " are the non-defected thresholds (defect-less threshold) in row and column segments. " $\mathrm{L}_{i}$ " shows the non-similarity amount between " $\mathrm{i}_{\mathrm{th}}$ " window and Basic vector.

\section{Testing Step}

In this step, first the test image is divided into windows of sizes $\mathrm{W} \times \mathrm{W}$ pixels. Then, for each window, the rows (column) feature vector is extracted. After that, for each of these windows the log-likelihood ratio is computed (Eq.15, Eq.16).

$\mathbf{D}_{\mathrm{xi}}=$

$\left(\mathrm{R}_{\mathrm{xi}}, \mathrm{M}_{\mathrm{x}}\right)=\sum_{\mathrm{k}=1}^{\mathrm{L}+1} \mathrm{R}_{\mathrm{kxi}} \log \left(\frac{\mathrm{R}_{\mathrm{kxi}}}{\mathrm{M}_{\mathrm{xk}}}\right) \mathrm{i}=1,2, \ldots, \mathrm{N}(15)$

$\mathbf{D}_{\mathrm{yi}}=$

$\left(\mathrm{R}_{\mathrm{yi}}, \mathrm{M}_{\mathrm{y}}\right)=\sum_{\mathrm{k}=1}^{\mathrm{L}+1} \mathrm{R}_{\mathrm{kyi}} \log \left(\frac{\mathrm{R}_{\mathrm{kyi}}}{\mathrm{M}_{\mathrm{yk}}}\right) \mathrm{i}=1,2, \ldots, \mathrm{N}(16)$

Where " $R_{i}$ " is the feature vector of row (column) process which is computed for " $i_{t h}$ " window of testing image. " $M_{x}$ " and " $\mathrm{M}_{\mathrm{y}}$ " are the basic vectors. Also, $\mathrm{N}$ shows the total number of windows and " $k$ " represents the " $k_{t h} "$ dimension of the feature vector.

The detection accuracy amount of proposed approach is related to the size of windows. There are two important points to determining the optimum size of windows as following: I. The larger the size of windows considered, the more accurate values would be obtained for feature vectors which are extracted for that window. But the larger the size of windows considered the detection rate would decrease for small defects. II. The numbers of operators which are applied on each window are related to the size of window and the length of row (column) segments. Tajeripour et al.(2008) suggest that the total number of applied operators must be larger than a threshold. In this respect, if the size of windows be $\mathrm{W} \times \mathrm{W}$ and the length of segments be $\mathrm{L}$ pixels, so the number of all row and column operators that are applied on each window is equal to Eq.17.

$$
2 \times[\mathrm{W} \times(\mathrm{W}-\mathrm{L}+1)]
$$

For example, if the operators threshold considered to be 120, consequently:

$$
2 \mathrm{~W}^{2}-2 \mathrm{WL}+2 \mathrm{~W}-120>0
$$

After computing non-similarity ratio between test window vectors and basic vectors, for each window, if any of these ratios is greater than the corresponding threshold, the window is declared as the defected window. It is shown in Eq.19.

$$
\begin{aligned}
& \mathrm{i}_{\text {th }} \text { Window }= \\
& \begin{cases}\text { Defected } & \text { if } \mathrm{D}_{\mathrm{xi}}>\mathrm{T}_{\mathrm{x}} \text { or } \mathrm{D}_{\mathrm{yi}}>\mathrm{T}_{\mathrm{y}} \\
\text { Non }- \text { Defected } & \text { otherwise }\end{cases}
\end{aligned}
$$

Output of the proposed approach is called defect pattern which is a binary image. Black pixels in the defect pattern represent defect-less areas of the surface and white pixels represent defected areas.

\section{Multi Resolution Analysis}

The proposed approach is a multi resolution method. So, the results of choosing the different size of segments can be mixed by using the following equations, and it can be used for detecting the abnormalities in the surfaces. It is shown in the following equations.

$$
\begin{aligned}
& \mathrm{L}_{\mathrm{xi}}^{\mathrm{Z}}=\sum_{\mathrm{z}=1}^{\mathrm{Z}} \mathrm{L}_{\mathrm{xi}}\left(\mathrm{R}_{\mathrm{xi}}^{\mathrm{z}}, \mathrm{M}_{\mathrm{x}}^{\mathrm{z}}\right) \\
& \mathrm{L}_{\mathrm{yi}}^{\mathrm{Z}}=\sum_{\mathrm{z}=1}^{\mathrm{Z}} \mathrm{L}_{\mathrm{xi}}\left(\mathrm{R}_{\mathrm{yi}}^{\mathrm{z}}, \mathrm{M}_{\mathrm{y}}^{\mathrm{z}}\right)
\end{aligned}
$$

Where, $Z$ is the number of segments different sizes and "i" corresponds to the " $\mathrm{i}_{\mathrm{th}}$ " windows.

\section{Experimental Results}

In this paper, a general approach was proposed for detecting the defects in the surfaces. In order to evaluate performance of the proposed approach, first 120 images were taken from 2 two different groups of surfaces such as "architectonic stone", and "patterned fabric textile".

For stone group, 3 categories of stones called "NonWavy Travertine", "Hatchet" and "Orange Travertine" is considered and 20 images were taken by a digital camera with resolution of $0.2 \mathrm{~mm} /$ pixel for each category. For fabric group, 3 categories of patterned fabric textiles called "Dot- Pattern", "Star- Pattern" and "Box- Pattern" is considered and 20 images were provided for each category by Department of electronic and electric of Hong-Kong University.

Detection rate is one of the popular criteria for measuring the performance of defect detection approaches (Bodnarova et al., 2000; Tajeripour et al., 2008). It is shown in Eq.22. So, the proposed approach was applied on the images and the detection rate was

Table 1. The detection rate, Specificity and Sensitivity of applying proposed approach on stone surface

\begin{tabular}{|l|c|c|c|}
\hline \multicolumn{4}{|c|}{ applying proposed approach on stone surface } \\
\hline Criteria Stone & $\begin{array}{c}\text { Creamy } \\
\text { Travertine }\end{array}$ & Hatchet & $\begin{array}{c}\text { Orange } \\
\text { Travertine }\end{array}$ \\
\hline Detection Accuracy & 95,6 & 96.2 & 95.7 \\
\hline Sensitivity & 93.6 & 94.4 & 93.9 \\
\hline Specificity & 96.8 & 96.7 & 96.8 \\
\hline
\end{tabular}

measured for each defect pattern. The average of detection rates in stone categories is shown in the second row of Table 1 and the average of detection rate in fabric categories is shown in the second row of Table 2. To apply the proposed approach, different sizes were 
tested for windowing, and the sizes $16 \times 16$ provided maximum detection rate in both of groups.

Detection Rate $=100 \times \frac{N_{R N D}+N_{R D}}{N_{\text {total }}}$

In Eq.22, $\mathrm{N}_{\mathrm{RD}}$ means the number of windows that were really defected and also were detected as defected window by the proposed approach. $\mathrm{N}_{\mathrm{RND}}$ means the number of windows which were really defect-less and also were detected as defect-less window. To measuring the detection rate, the defect pattern was divided to nonoverlap windows by the sizes of $16 \times 16$ pixels. After that, each window that has at least one defected pixel was counted as a defected window.

Fig.4. Determining result by defect detected image

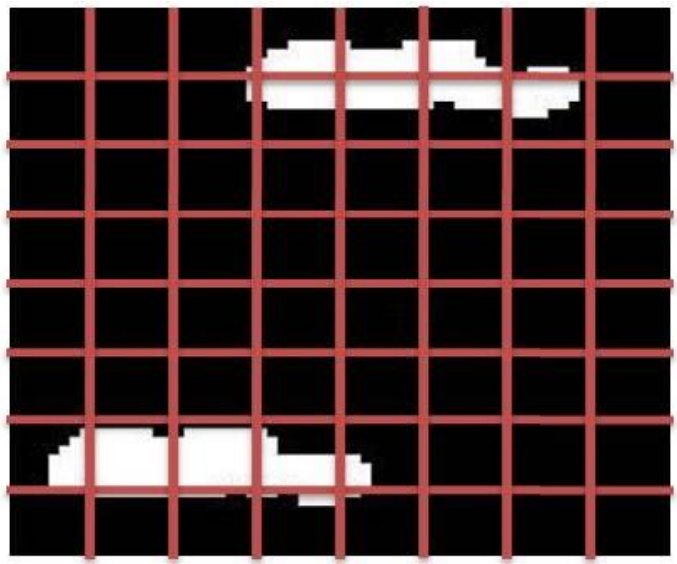

In Fig.4 a defect pattern is shown where the first window (first column and first row) is categorized as nondefected window and the fourth window (fourth column and first row) is categorized as defected window.

To evaluate performance more accurate, the specificity (Eq.23) and sensitivity (Eq.24) were computed for all of the defect patterns. Average of these is shown in the second and third rows of the Tables 1 , and 2 .

$$
\begin{aligned}
& \text { Specificity }=\frac{T N}{T N+F P} \\
& \text { Sensitivity }=\frac{T P}{T P+F N}
\end{aligned}
$$

Where, TP, TN, FP and FN means true positive, true negative, false positive and false negative.

\section{Detection Accuracy Comparison}

In order to compare the performance of 2DLBP and 1DLBP, 2DLBP was applied on all of the stone images (Tajeripour \& Fekri-Ershad, 2012). After that, detection rate were measured for all of the defect patterns. Averages of these are shown in Table 3. For applying the $2 \mathrm{DLBP}$, different neighborhoods sizes of neighbors $(3 \times 3$, $5 \times 5$ and $7 \times 7$ ) were used.

In order to compare 2DLBP and 1DLBP results, some of the visual results are shown in Fig.5.
Table 2. The detection rate, Specificity and Sensitivity of applying proposed approach on fabric surface

\begin{tabular}{|l|c|c|c|}
\hline $\begin{array}{c}\text { Ratterned Fabric } \\
\text { Criteria }\end{array}$ & $\begin{array}{c}\text { Dot } \\
\text { Pattern }\end{array}$ & $\begin{array}{c}\text { Box } \\
\text { Pattern }\end{array}$ & $\begin{array}{c}\text { Star } \\
\text { Pattern }\end{array}$ \\
\hline Detection Accuracy & 96,6 & 97.8 & 96.8 \\
\hline Sensitivity & 93.2 & 94.4 & 96.2 \\
\hline Specificity & 97.9 & 98.3 & 97.5 \\
\hline
\end{tabular}

\section{Computational Complexity Comparison}

In order to compare the performance of 1DLBP with other versions of LBP, the computational complexity of the 1DLBP is compared with 2DLBP. In this respect, the number of operations which are required for processing a test sample is considered.

Total number of detection windows is independent of texture analysis operator type so, it is same in 2DLBP and 1DLBP process. For example, if the size of test sample is $256 \times 256$, the size of detection window is $16 \times 16$, and the overlapping step between detection windows is 8 pixels, total number of detection windows will be 961 .

Table 3. The average of detection rate of applying 2DLBP on

\begin{tabular}{|c|c|c|c|c|}
\hline Features & 2DLBP & $\begin{array}{l}\text { Creamy } \\
\text { Travertine }\end{array}$ & Hatchet & $\begin{array}{l}\text { Orange } \\
\text { Travertine }\end{array}$ \\
\hline 10 & 8,3 & 88,68 & 91.64 & 90.02 \\
\hline 18 & 16,5 & 93,67 & 92.27 & 94.43 \\
\hline 26 & 24,7 & 90.05 & 91.32 & 95.43 \\
\hline $10+18$ & $8,3+16,5$ & 93.22 & 90.53 & 91.14 \\
\hline $10+26$ & $8,3+24,7$ & 89.60 & 89.03 & 90.75 \\
\hline $18+26$ & $16,5+24,7$ & 89.44 & 94.11 & 92.46 \\
\hline $10+18+26$ & $8,3+16,5+24,7$ & 85.54 & 87.73 & 91.37 \\
\hline
\end{tabular}
stone surface

Table 4. Number of different operations which are required to process a test sample using 1DLBP and 2DLBP.

\begin{tabular}{|l|c|c|}
\hline \multicolumn{1}{|c|}{ Operator } & 1DLBP & 2DLBP \\
\hline Comparison & $\begin{array}{c}{[\mathrm{W} \times(\mathrm{W}-\mathrm{L}+1)]} \\
\times 2 \times(\mathrm{L}-1)\end{array}$ & $\begin{array}{c}(\mathrm{W}-\mathrm{R}+1) \times \\
(\mathrm{W}-\mathrm{R}+1) \times \mathrm{P}\end{array}$ \\
\hline Multiplication & $2 \times(\mathrm{L}+1)$ & $\mathrm{P}+2$ \\
\hline Division & $2 \times(\mathrm{L}+1)$ & $\mathrm{P}+2$ \\
\hline Addition & $2 \times(\mathrm{L}-1)$ & $\mathrm{P}$ \\
\hline Subtraction & 0 & 0 \\
\hline
\end{tabular}

In each detection window, for applying $2 \mathrm{DLBP}_{\mathrm{P}, \mathrm{R}}$, the number of required operations are shown in the second column of table6. Numbers of required operations for applying $1 \mathrm{DLBP}_{\mathrm{L}}$ in each detection windows are shown in the first column of Table. 4. Where, the size of detection window is $\mathrm{W} \times \mathrm{W}$.

In the result part, to evaluate the performance, the length of each row (column) segment is considered 8 pixels. In each detection window, the number of required operations of $\mathrm{LBP}_{8}$ is compared with $\mathrm{LBP}_{\mathrm{P}, \mathrm{R}}(\mathrm{R}=3,5$, \& 7 ), it is shown in Tables $5 \& 6$. Where, in the Table 5 , the 
Table 5. Number of different operations which are required to process a test sample using 1DLBP and 2DLBP

\begin{tabular}{|c|c|c|c|}
\hline Operator & $\begin{array}{c}\text { Compariso } \\
\mathrm{n}\end{array}$ & $\begin{array}{l}\text { Multiplication } \\
\text { (Division) }\end{array}$ & Addition \\
\hline 1DLBP 8 & 2016 & 18 & 14 \\
\hline 2 DLBP $_{8,3}$ & 1568 & 10 & 8 \\
\hline $\operatorname{2DLBP}_{16,5}$ & 2304 & 18 & 16 \\
\hline $2 \mathrm{DLBP}_{24,7}$ & 2400 & 26 & 24 \\
\hline $\begin{array}{l}\operatorname{2DLBP}_{8,3}+ \\
2_{2 L_{16,5}}\end{array}$ & 3872 & 28 & 24 \\
\hline $\begin{array}{l}\operatorname{2DLBP}_{8,3^{+}} \\
\text {2DLBP }_{24,7}\end{array}$ & 3968 & 36 & 32 \\
\hline $\begin{array}{l}\operatorname{2DLBP}_{16,5^{+}} \\
\text {2DLBP }_{24,7} \\
\end{array}$ & 4704 & 44 & 40 \\
\hline $\begin{array}{c}\operatorname{2DLBP}_{8,3^{+}} \\
\operatorname{2DLBP}_{16,5^{+}} \\
2^{2} \operatorname{LBP}_{24,7}\end{array}$ & 6272 & 54 & 48 \\
\hline
\end{tabular}

size of detection window is considered $16 \times 16$ and in it is considered $25 \times 25$ in Table 6 . Where, the total number of other required operations such as subtraction and square root is zero. Also in Tables 6 and 7 the total number of required multiplications and divisions is equal. As it was
Vol. 5 No. 8 (August 2012) ISSN: 0974- 6846

Table 6. Number of different operations which are required to process a test sample using $1 D L B P$ and $2 D L B P$

\begin{tabular}{|c|c|c|c|}
\hline Operator & Comparison & $\begin{array}{l}\text { Multiplication } \\
\text { (Division) }\end{array}$ & Addition \\
\hline $1 \mathrm{DLBP}_{8}$ & 6300 & 18 & 14 \\
\hline 2 DLBP $_{8,3}$ & 4232 & 10 & 8 \\
\hline 2 DLBP $_{16,5}$ & 7056 & 18 & 16 \\
\hline $2 \operatorname{DLBP}_{24,7}$ & 8664 & 26 & 24 \\
\hline $\begin{array}{l}\operatorname{2DLBP}_{8,3}+ \\
2_{16,5} \operatorname{DLP}_{16,5}\end{array}$ & 11288 & 28 & 24 \\
\hline $\begin{array}{l}\operatorname{2DLBP}_{8,3}+ \\
2_{2 L B P}{ }_{24,7}\end{array}$ & 12896 & 36 & 32 \\
\hline $\begin{array}{c}\operatorname{2DLBP}_{16,5^{+}} \\
\operatorname{2DLBP}_{24,7}\end{array}$ & 15720 & 44 & 40 \\
\hline $\begin{array}{c}\operatorname{2DLBP}_{8,3^{+}} \\
2^{+D_{B P}}{ }_{16,5^{+}} \\
\operatorname{LLBP}_{24,7}\end{array}$ & 19952 & 54 & 48 \\
\hline
\end{tabular}

seen in Tables 8 and 9, the computational complexity of $1 \mathrm{DLBP}_{8}$ is nearly as the same as $2 \mathrm{DLBP}_{16,5}$, but the detection rate of 1DLBP is too more. Also, the computational complexity of proposed 1DLBP is less than other 2DLBP operators.
Fig.5 (a) Original Image of Dot-pattern Fabric Textile

(b) Defect detected of (a) by $2 D L B P 7 \times 7$ (c) defect detected of (a) by $1 D L B P$ with $L=8$

(d) Original Image of Star-Pattern Fabric Textile (e) defect detected of (d) by 2DLBP $5 \times 5$ (f) defect detected of (d) by $1 D L B P$ with $L=8$

(g) Original Image of Non-wavy Creamy Travertine Stone (h) defect detected of $(\mathrm{g})$ by $2 D L B P 3 \times 3 \& 7 \times 7$ (i) defect detected of $(\mathrm{g})$ by $1 D L B P$ with $L=8$
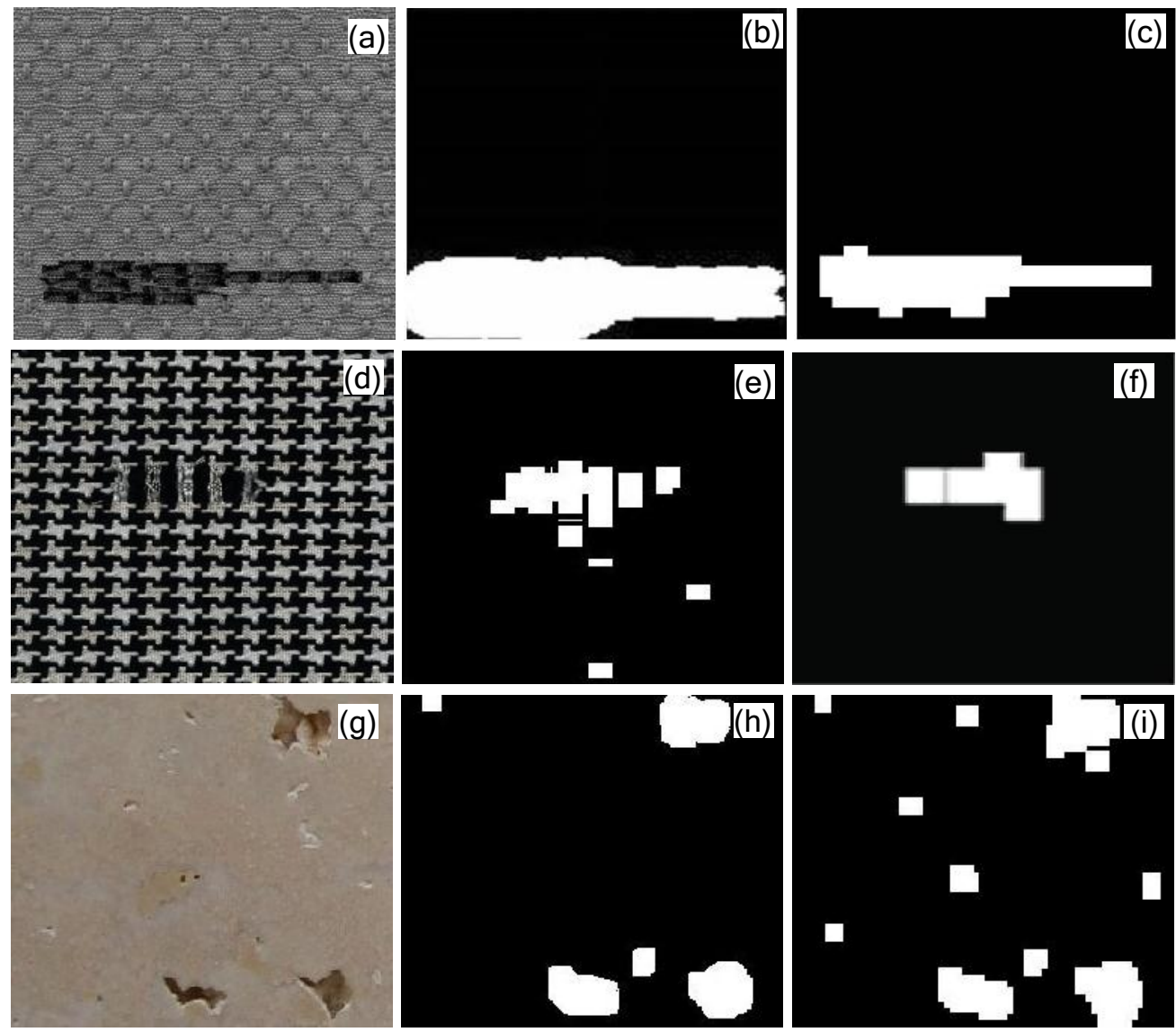

Research article

CIndian Society for Education and Environment (iSee)

\section{Conclusion}

The goal of this article was to propose an accurate, general, and online approach for detecting the defects and abnormalities in surfaces. In order to satisfy the goals of paper, a novel approach is proposed based on modified LBP called one dimensional local binary patterns. The experimental results have showed that the proposed approach has a high detection rate for different surfaces such as stone, paper and textile. Some other advantages of this approach are as follows:

I) 1DLBP operator that proposed in this paper has less computational complexity than previous versions of LBP such as 2DLBP. II) The proposed approach has low sensitivity to noise as a result of windowing technique and considering the relation between each pixel and its neighbors. III) High detection rate of the proposed defect detection approach (section5) using 2DLBP as texture analysis operator shows the suitability of proposed defect detection approach with other feature operators. IV) The 
proposed approach is a general one for two class classification problems. So it can be used for every defect detection cases such as metal papers, ceramics and etc. V) In this paper, a novel feature vector is described that can be used for other image processing cases to analyze and classify the textures. VI) Online ability is unique advantage of proposed approach comparing other defect detection methods.

\section{References}

1. Alimohamdi $\mathrm{H}$ and Ahmady A (2006) Detecting skin defect of fruits using optimal Gabor wavelet filter. In: Proc. of Intl. Conf. on Digital Image Processing, pp. 402-406.

2. Bodnarova A, Bennamoun M and Kubik K (2000) Suitability analysis of techniques for flaw detection in textiles using texture analysis. Pattern Anal. \& Appl. 3. 254-266.

3. Bua HG, Wanga J and Huanga X (2009) Fabric defect detection based on multiple fractal features and support vector data description. Eng. Appl. Artificial Intelligence. 22(2), 224-235.

4. Ghazini M, Monadjemi A and Jamshidi K (2009) Defect detection of tiles using 2D Wavelet transform and statistical features. World Acad. Sci., Eng. \& Technol. 49, 901-904.

5. Henry Y, Grantham K and Nelson H (2010) Ellipsoidal decision regions for motif-based patterned fabric defect detection. Pattern Recognition. 43(6), 21322144.

6. Lin $\mathrm{CH}$ and Yu-Yeh C (2009) Texture defect detection system with image deflection compensation. WSEAS Transact. on Compu. 8(9), 1575-1586.

7. Ojala T, Maeenpaeae T and Pietikaeinen M (2000) Texture Classification by Multi Predicate Local Binary Pattern Operators. In: Proc. 15th Intl. Conf. on Pattern Recognition. 3, 951-954.

8. Ojala T, Pietikainen M and Maenpaa T (2002) Multi resolution gray-scale and rotation invariant texture classification with local binary patterns. IEEE Transact. on Pattern Analysis \& Machine Intelligence, 24(7), 971-987.

9. Pietikäinen M, Ojala T and Xu Z (2000) RotationInvariant texture classification using feature distributions. Pattern Recognition. 33, 43-52.

10.Tajeripour $\mathrm{F}$ and Fekri-Ershad SH (2012) Porosity detection by using improved local binary pattern. In: Proc. of the 11th WSEAS Intl. Conf. on Signal Processing, Robotics and Automation (ISPRA '12), 1, 116-121.

11.Tajeripour F, Kabir E and Sheikhi A (2008) Fabric defect detection using modified local binary patterns. EURASIP J. on Adv. in Signal Processing. 1, 1-12.

12.Tolba AS (2011) Fast defect detection in homogeneous flat surface products. Expert Systems with Appl.: An Intl. J. 38(10), 12339-12347.
13.Tsai D, Lin C and Huang K (2005) Defect detection in coloured texture surfaces using Gabor filters. Imaging Sci. J. 53(1), 27-37.

14.Wen W and Xia A (1999) Verifying edges for visual inspection purposes. Pattern Recognition Lett. 20, 315-328.

15.Xie $X$ (2008) A review of recent advances in surface defect detection using texture analysis. Electronic Lett. on Computer Vision \& Image Analysis.7(3), 1-22. 